\title{
STRATEGIES TO AVOID OPIOIDS AND SPARE THE DIAPHRAGM IN DAY-CASE SHOULDER SURGERY: CASE STUDIES
}

\author{
Bruno Lima ${ }^{1}$, Pedro Moreira ${ }^{1}$, Carolina Martins ${ }^{2}$, Paulo Fragoso ${ }^{1}$ \\ 1 - Anaesthesiology Department, Hospital de Braga. Braga, Portugal \\ 2 - Anaesthesiology Department. Centro Hospitalar de Lisboa Ocidental. Lisboa, Portugal
}

\section{INTRODUCTION}

Shoulder surgery is associated with significant postoperative pain. Interscalene block (ISB) remains the cornerstone in shoulder surgery, but it may result in phrenic nerve block in up to $100 \%$ of the cases.

Opioid use is limited by its side-effects and it is best avoided in the outpatient setting. This prompts for different approaches to the anaesthetic technique.

Opioid-free anaesthesia combined with diaphragm-sparing nerve blocks can minimize complications providing effective analgesia, enhanced recovery and a better outcome.

Our purpose was to evaluate the efficacy and feasibility of an anaesthetic protocol that would meet these requirements.

\section{CASE STUDIES}

-Five patients, 34 to 78 years-old, ASA II

-Surgical procedure: shoulder arthroscopy - rotator cuff repair surgery

-Anaesthetic technique: Superficial cervical plexus block $(5 \mathrm{~mL})$, low-volume "extrafascial" interscalenic block, lateral to C5-C6 $(5 \mathrm{~mL})$ and posterior and lateral cords block at infraclavicular level $(5+5 \mathrm{~mL})$, with a mixture of $0,5 \%$ Lidocaine, $0,5 \%$ Ropivacaine and Dexamethasone $4 \mathrm{mg}$, followed by LMA ProSeal ${ }^{\circledR}$ general anaesthesia, induction with propofol and maintenance with Sevoflurane/O2/air mixture

\section{A}

SCM

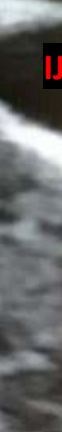

M
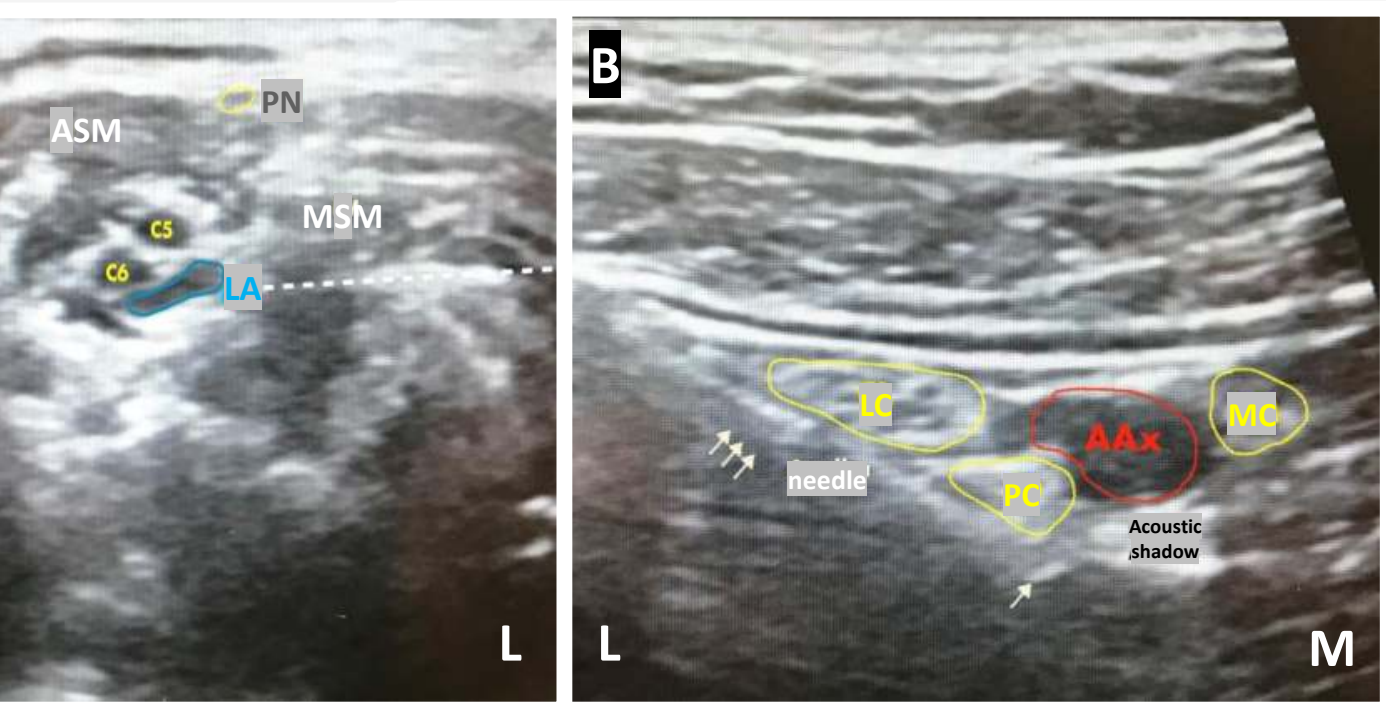

Figure 1: A- Ultrasound guided "extrafascial" interscalenic block, lateral to C5-C6; B- Ultrasound guided posterior and lateral cords block at infraclavicular level

AAX-Axillary artery ASM- Anterior scalene muscle CA- Carotid artery C5, C6- cervical vertebrae 5 and $6 \mathrm{IJV}$ - internal jugular vein M- medial MSM- middle scalene muscle MC- medial cord LC- lateral cord PC- posterior cord PN- Phrenic nerve SCMsternocleidomastoid muscle L- lateral LA- Local anesthetic

All patients were monitored according to ASA standards plus monitoring bispectral index, $\mathrm{BIS}^{\circledast}$ (to a target 40 to 60 ) and cerebral oxygenation monitoring INVOS ${ }^{\circledR}$ ( to a target rSO2 variation $<20 \%$ of baseline)
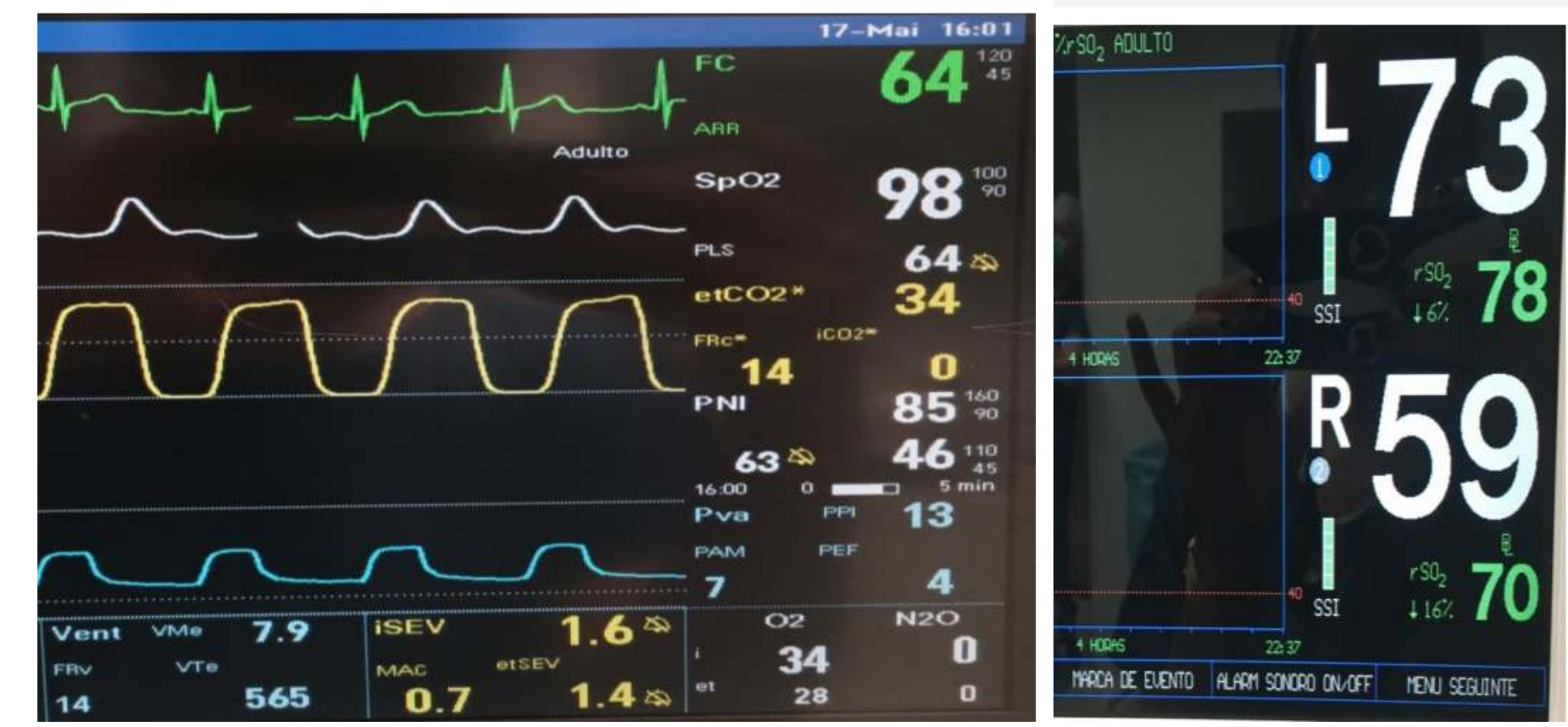

Figure 2: One of the patient's monitoring

Positioning: beach chair positioning
Intra-operative: all patients were hemodynamically stable with optimal surgical conditions. Multimodal analgesia was provided with intravenous paracetamol and NSAID's

Postoperative:

All

patients reported mild to no pain (VAS 0-3), no nausea or vomiting. After recovery, at PACU, diaphragmatic function was evaluated with ultrasound. No phrenic block was registered.

\section{First post-op day}

\section{RESULTS}

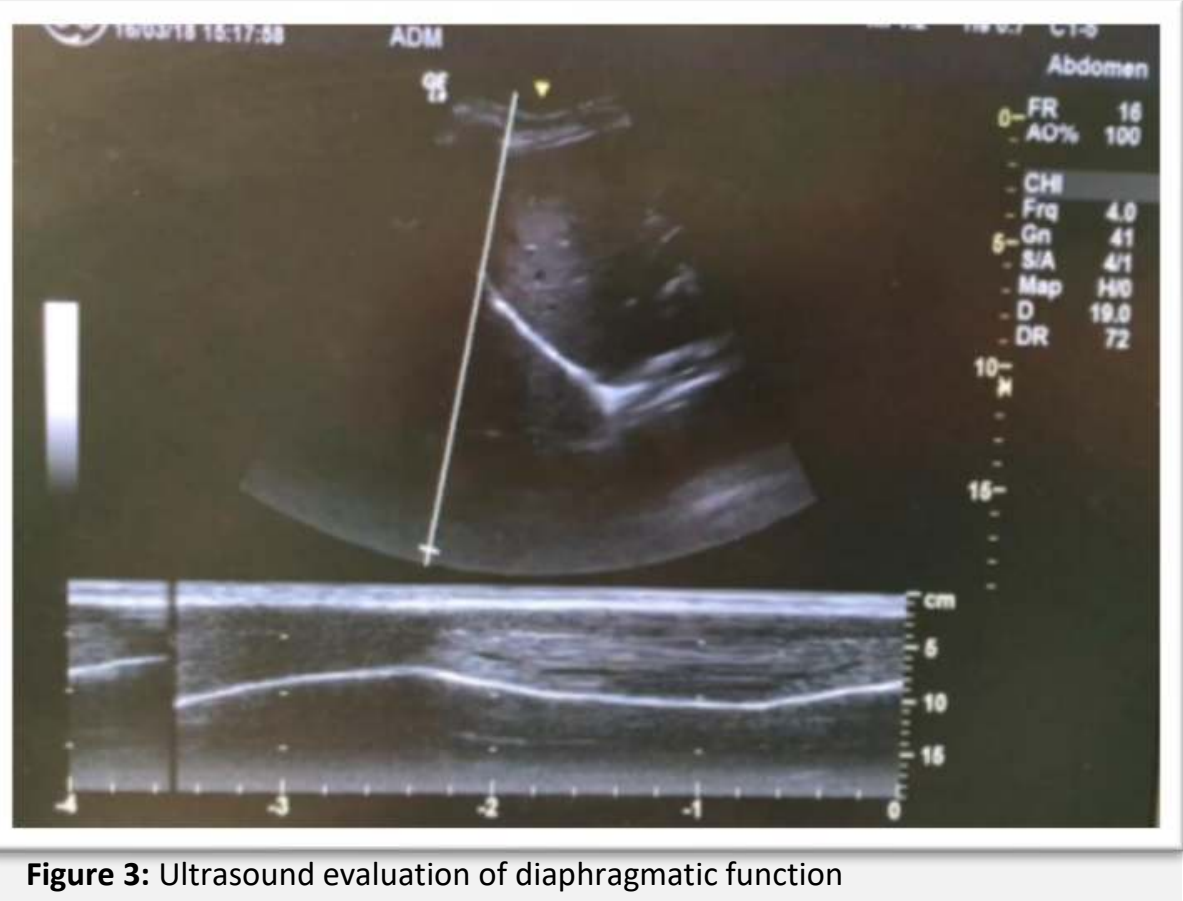

FOLLOW-UP
All patients were discharged the day after, with no need for rescue analgesia, except one patient that needed per os paracetamol plus tramadol for moderate pain.

Consultation by a physiatrician was provided before discharge.

\section{4 hours phone-call}

4 patients answered and reported mild or no pain (VAS $0-3$ )

$8^{\text {th }}$ day post-op

None of the patients needed rescue analgesia. No other complaints were registered.

\section{DISCUSSION AND CONCLUSION}

The anaesthetic protocol employed in these cases allowed adequate pain control with no opioid side-effects, providing adequate surgical conditions and preserving brain perfusion, contributing to overall patient satisfaction and early recovery

Diaphragm-sparing nerve blocks with adequate anaesthetic coverage helped to minimize complications, maintaining respiratory performance. This proved to be a valid strategy in outpatient setting. Nevertheless, further studies, enrolling more patients are necessary to establish a definitive protocol.

\section{REFERENCES}

El-Boghdadly K, et al. Phrenic Nerve Palsy and Regional Anesthesia for Shoulder Surgery: Anatomical, Physiologic, and Clinical Considerations. Anesthesiology. 2017 Jul; 127(1):173-191.

McGoldrick KE. Day-case Shoulder Surgery: Anesthetic Challenges. Ambulatory Surgery, 2012; 17(3): 47-49.

Mulier J. Anestesia libre de opioides: ¿un cambio de paradigma? Rev Esp Anestesiol Reanim. 2017;64:427-430 Palhais $\mathrm{N}$, et al. Extrafascial injection for interscalene brachial plexus block reduces respiratory complications compared with a conventional intrafascial injection: a randomized, controlled, double-blind trial. Br J Anaesth. 2016 Apr; 116(4): 531-537.

Tran DQ, et al. Diaphragm-Sparing Nerve Blocks for Shoulder Surgery. Reg Anesth Pain Med. 2017 Jan/Feb; 42(1):32-38. 